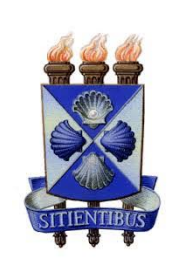

\title{
Estudo Ab-initio das propriedades eletrônicas e ópticas dos compostos TIN e TIAs submetidos à pressão hidrostática
}

\author{
$\underline{\text { Joseane Santos Almeida }}^{1}$ e Álvaro Santos Alves ${ }^{2}$ \\ ${ }^{1}$ Bolsista PROBIC/UEFS, Graduanda em Física, Universidade Estadual de Feira de Santana, e-mail: \\ joseanealmeida107@gmail.com \\ ${ }^{2}$ Orientador, Departamento de Física, Universidade Estadual de Feira de Santana, e-mail: asa@ uefs.br
}

PALAVRAS-CHAVE: TIN; TIAs; DFT.

\section{INTRODUÇÃO}

Os compostos III-V tem sido amplamente estudados devido à propriedades que enquadramse para fabricação de dispositivos eletrônicos e optoeletrônicos, como diodos emissores de luz e fotodectores (Mazouz, 2010). Nesse contexto, as pesquisas com os compostos Tálio-V, tais como TIN,TIP, TlAs, TISb e TlBi, têm apresentado resultados relevantes, indicando a possibilidade desses materiais serem utilizados pela indústria de semicondutores (Saidi-Houat, 2007;Elhassasna, 2019). Alguns estudos teóricos indicaram que o tálio apresenta fase supercondutora quando submetido à pressão hidrostática (Komsilp, 2016;Komsilp, 2017) e também configura uma composição importante nos óxidos supercondutores de alta temperatura crítica (Lazarev, 1963;Greenblatt, 1990; Hatton, 1955). Em simulações de pressão nula e sem considerar o acoplamento spin-órbita(SOC), foi mostrado que o TIN exibe comportamento metálico (Shi, 2010). Entretanto, ao acrescentar o SOC, o TIN é um semicondutor de gap pequeno entre $0.03 \mathrm{eV}$ a $0.10 \mathrm{eV}$ (Farzan,2016;Winiarski, 2015). Ademais, Sheng et al (2014), apresentaram resultados interessantes ao analisar o efeito da hibridização entre os orbitais N-2p e Tl-5d e o ajuste do acoplamento spin-órbita efetivo. Ao tornar negativo o SOC efetivo, o TIN se comporta como isolante topológico. Ou ainda, ajustando o SOC para positivo, induz-se uma transição para um semimetal de Dirac. Saidi(2007) et al, utilizando o método full-potential linearized augmented plane wave (FP-LAPW), computaram a energia total de diferentes compostos Tl-V em seis distintas estruturas cristalinas. Nesse trabalho, o arseneto de tálio (TlAs) apresenta a fase zinc-blende. Ao submetê-lo à pressão hidrostática, ocorre uma transição de fase para a estrutura rock-salt, em torno de 5.01 GPa (Shi,2010). No que tange as propriedades eletrônicas, o TlAs apresenta um caráter metálico (Mazouz, 2010). Mediante o levantamento bibliográfico, não foi encontrado artigos que incluam spin-órbita no arseneto de tálio. Assim, acrescentá-lo pode trazer resultados não apresentados na literatura. No presente trabalho, estudamos as propriedades estruturais e eletrônicas do TIN e TlAs na fase zinc-blende com e sem acoplamento spin-órbita, além da estrutura de bandas do nitreto de tálio sob pressão hidrostática. 


\section{METODOLOGIA}

Para avaliar as propriedades estruturais e eletrônicas do TIN e TlAs, utilizou-se a Teoria funcional da Densidade (DFT)(Hohenberg, 1964), que consiste em solucionar as equações autoconsistentes de Khon-Sham (Khon, 1965). Ao escolher uma base para expandir os orbitais, o problema se resume em diagonalizar matrizes. A função base empregada foi projected augmented wave (PAW)(Blöchl,1994), implementado pelo programa QUANTUM ESPRESSO (GIANNOZI,2009). O potenciais de troca e correlação utilizado se denomina Generalized Gradiente Approximation, parametrizado por Perdew e Burke (GGA-PBE)(Perdew, 1996). Limitamos o número de funções base ao escolher o máximo vetor de onda $G_{\max }$ no espaço recíproco. Além disso, escolhemos o número de pontos $k$ usados em integrações na zona de Brillouin, em que a malha é gerada pelo método Monkhorst-Pack (Monkhorst, 1976). O critério de convergência utilizado para a força foi de $10^{-4} \mathrm{Ry} /$ bohr e $10^{-5} \mathrm{Ry} / \mathrm{bohr}$ para a energia total. Tal energia convergiu bem em relação à temperatura eletrônica (Marzari-Vanderbilt-DeVita-Payne cold smearing) com o valor de 0.0025 Ry para o TIN e 0.001 para o TlAs. Posteriormente, efetuou-se a otimização da estrutura cristalina. As energias totais e volumes foram fitados pela equação de estado Birch-Murnaghan, onde o volume de equilíbro $V_{o}$, o bulk modulus $B_{o}$ e sua derivada $B_{o}^{\prime}$ foram determinados para cada composto. Ademais, foram encontradas as posições atômicas relaxadas, em que as forças sobre os átomos são nulas. Finalmente, a estrutura de bandas e a densidade de estados (DOS) permitiu analisar as propriedades eletrônicos do TlN e TlAs.

\section{RESULTADOS E/OU DISCUSSÃO (ou Análise e discussão dos resultados)}

No QUANTUM ESPRESSO, o critério para encontrar um número adequado de ondas planas e vetores recíprocos consiste em avaliar a diferença de energia por átomo. Os valores escolhidos são tais que as diferenças se localizem no intervalo de apenas 0.0004 Ry. Para o TIN, com e sem SOC, a energia total converge bem com uma energia de corte de 80 Ry e uma malha $8 \times 8 \times 8$ de vetores de onda $\mathrm{k}$. Os resultados foram os mesmos para o TlAs. O próximo passo constituiu a otimização da estrutura. Após calcular a energia total para diferentes valores do volume, o fit desse conjunto de dados com a equação de Birch-Murnaghan apresentou resultados em concordância com a literatura já existente(Farzan, 2016; Saidi-Houat, 2009; Shi, 2010; Mazouz,2010). Segue o resumo na tabela abaixo.

\begin{tabular}{|c|c|c|c|c|}
\hline & TIN-Sem SOC & TIN-Com SOC & TlAs-Sem Soc & TlAs-Com Soc \\
\hline a (bohr) & 9.9745 & 9.9474 & 12,0276 & 11,9833 \\
\hline$B_{o}(\mathrm{GPa})$ & 88,68 & 90,33 & 38,00 & 39,00 \\
\hline$B_{o}^{\prime}$ & 5,16 & 5,15 & 4,62 & 5,37 \\
\hline$E_{\min }(\mathrm{Ry})$ & $-864,859$ & $-872,02$ & $-1012,75$ & $-1020,09$ \\
\hline
\end{tabular}

Tabela 1: Parâmetros de rede do TIN e TlAs.

O TIN sem acoplamento spin-órbita apresenta uma estrutura de bandas com gap zero, em que as bandas de valência e condução se tocam no nível de fermi na direção do ponto $\Gamma$. A densidade de estados projetada demonstra que a banda de valência recebe maior contribuição do 
estado $2 p$ do nitrogênio e que na banda de condução predomina os orbitais $2 p$ do $\mathrm{N}$, 6 s e $6 \mathrm{p}$ do Tl.Ao acrescentar o SOC, o TIN apresenta um pequeno gap de $0,03 \mathrm{eV}$ em torno do ponto $\Gamma$, porém a densidade de estados varia pouco em torno do nível de fermi.
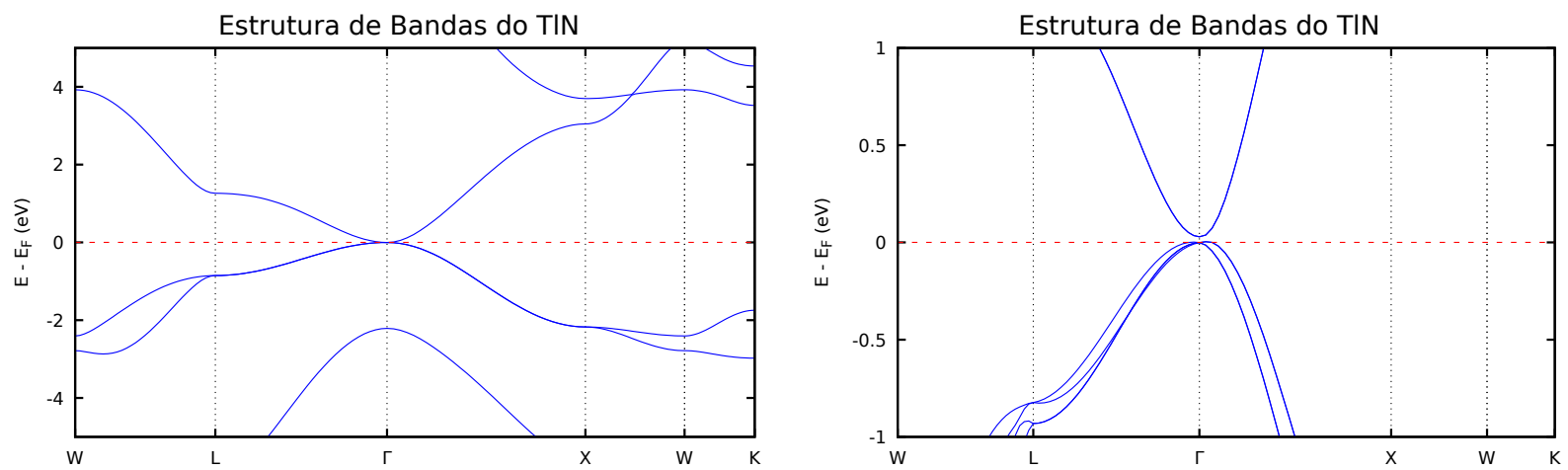

Figura 1: Estrutura de bandas do TIN próximo ao nível de Fermi sem e com SOC, da esquerda para direita.

A figura 2 exibe a estrutura de bandas do nitreto de tálio sem SOC quando submetido às pressões hidrostáticas de 0GPa e 19GPa. É possível notar que, ao aplicar pressão, os níveis de energia maiores que o nível de Fermi se deslocam para níveis acima. Enquanto isso, os níveis menores que a energia de fermi, exceto a terceira banda de valência no ponto $\Gamma$, diminuem. Entretanto, o gap permanece nulo e o composto mantém-se metálico. De forma geral, as contribuições dos orbitais em torno do nível de fermi no ponto gama diminuíram.
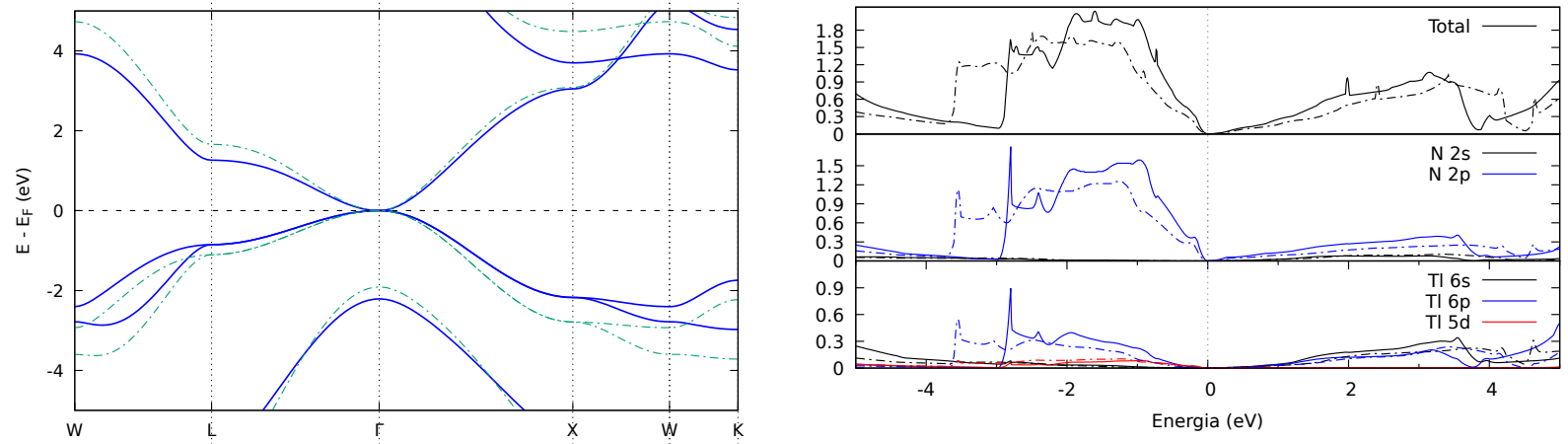

Figura 2: Estrutura de bandas e DOS do TIN sem SOC em 0GPa (linha cheia) e 19GPa (linha tracejada), da esquerda para direita.

O TlAs sem acoplamento spin-órbita possui um pequeno gap negativo e indireto. $\mathrm{O}$ máximo da banda de valência está no ponto $\Gamma$ com $0,0386 \mathrm{eV}$. O mínimo de condução é no ponto L, com -0,0815 eV. Analisando a densidade de estados projetada, vemos que os orbitais $4 \mathrm{p}$ do arsênio e $6 \mathrm{p}$ do tálio contribuem significativamente para a banda de valência e os orbitais $4 p$ do As e 6 s do Tl contribuem consideravelmente para a banda de condução. 

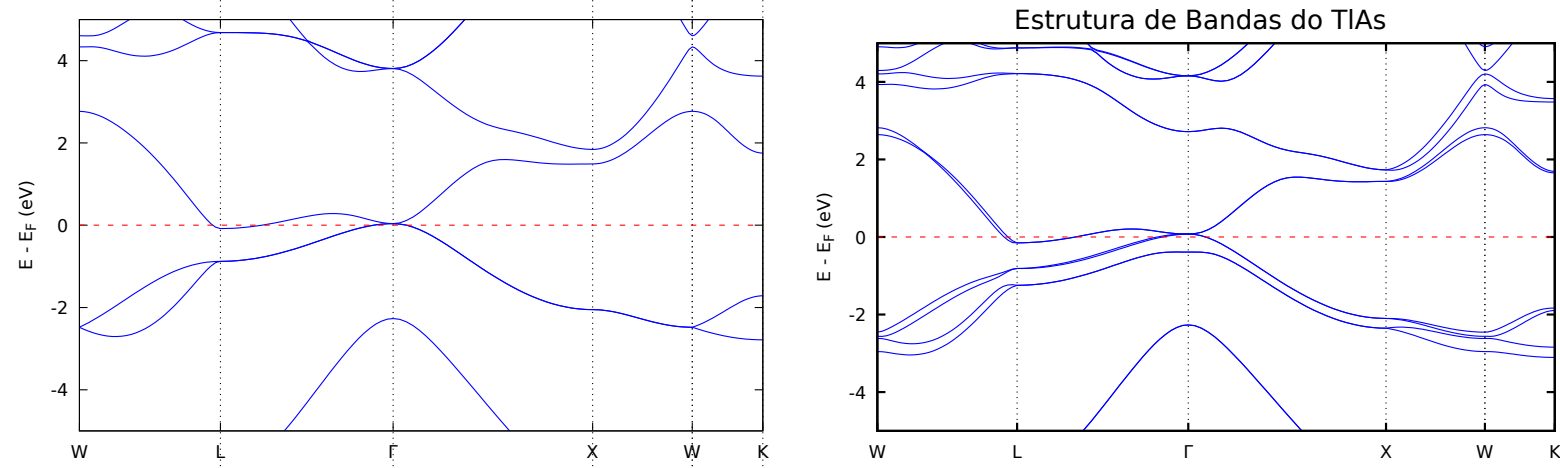

Figura 3: Estrutura de bandas do TlAs próximo ao nível de Fermi sem e com SOC, da esquerda para direita.

Ao acrescentar o acoplamento spin-órbita há uma quebra na degenerescência das bandas de valência. Entretanto, o SOC não modificou significativamente o gap negativo e indireto entre o ponto L e $\Gamma$.

\section{CONSIDERAÇÕES FINAIS (ou Conclusão)}

Nesse trabalho foi possível investigar as estruturais e eletrônicas do nitreto de tálio e arseneto de tálio na fase zinc-blende. Sem o acoplamento spin-órbita, concluímos que o composto TIN é metálico e o TlAs é um semi-metal, em que o orbital d do átomo de tálio contribui significativamente para as bandas em torno do nível de fermi no ponto gama. Além disso, a pressão não foi suficiente para induzir um gap no nitreto de tálio e as contribuições dos orbitais na densidade de estados pouco mudaram. Contudo, o SOC foi capaz de provocar o aparecimento de um pequeno gap, levando o composto TIN à semicondutor. Enquanto isso, o arseneto de tálio não modificou seu comportamento eletrônico ao incluir a interação spin-órbita. Os resultados estão de acordo com estudos teóricos anteriores e serão úteis para a continuidade do trabalho.

\section{REFERÊNCIAS}

MAZOUZ, et al.2010.First-principles study of lattice dynamics in thallium-V compounds. Superlattices Microstruct. 48, 560-568.

SAIDI-HOUAT, N. ZAOUI, A. FERHAT, M. 2007. Structural stability of thallium-v compounds. Journal of Physics: Condensed Matter, vol. 19, no. 10, p. 106221.

ELHASSASNA A., BECHIRI, A.2019. Electronic and Elastic Properties of TlX (X= N, P, As and $\mathrm{Sb})$ in Zinc-Blende Structure. Solid State Phenom. 297:82-94.

KOTMOOL, K. et al.2016. PNAS October 4, 113 (40) 11143-11147.

KOTMOOL, K. et al. 2017.Scientific Reports, vol 7, 42983.

LAZAREV, B.G., LAZAREVA, L.S., MAKAROV, V.I.1963. Some Singularities of the Behavior of the Superconducting Transition Temperature of Thallium Under Pressure. JETP, Vol. 17,No.2. GREENBLATT, M. et al.1990. Chemistry and superconductivity of Thallium-based cuprates. Studies of High Temperature Superconductors, ed Narlikar A (Nova Science, New Brunswick, NJ), pp 143-170. 
HATTON, J.1955. Effect of Pressure on the Superconducting Transition Temperature of Thallium. Phys. Rev. 100, No. 6.

SHENG, X. et al. 2014. Topological insulator to dirac semimetal transition driven by sign change of spin-orbit coupling in thallium nitride. Physical Review B, vol. 90, no. 24, p. 245308.

SHI, L. , DUAN Y., QIN, L. 2010. Structural phase transition, electronic and elastic properties in tlx $(x=n, p$, as) compounds: Pressure-induced effects. Computational Materials Science, vol. 50, no. 1, pp. 203-210.

FARZAN, et al. 2016. A comparison of the structural, electronic, optical and elastic properties of wurtzite, zinc-blende and rock salt tln: A dft study. Acta Physica Polonica, A., vol. 130, no. 3.

WINIARSKI. 2015. M. Winiarski, "The band-gap of tl-doped gallium nitride alloys," Computational Materials Science, vol. 108, pp. 14-16.

HOHENBERG, P. ; KHON, W. 1964. Inhomogeneous Electron Gas. Phys. Rev. 136, B864.

KHON, W. SHAM, L. J. 1965. Self-Consistent Equations Including Exchange and Correlation Effects. Phys. Rev. 140, A1133.

BlöCHL, P. E. 1994. Projector augmented-wave method. Physical Review B,50(24):17953-17979.

GIANNOZZI, P et al. 2009. QUANTUM ESPRESSO: a modular and open-source software project for quantum simulations of materials. J. Phys.: Condens. Matter. 21, 395502.

MONKHORST, J. H. PACK, J.D. 1976. Special points for Brillouin-zone integrations.Phys. Rev. B 13, 5188.

ANDERSEN, O. K. 1975. Linear methods in band theory. Physical Review B, vol. 12, no. 8, p. 3060, 1975.

GULANS, A. et al . 2014. exciting - a full-potential all-electron package implementing densityfunctional theory and many-body perturbation theory, J. Phys.: Condens. Matter 26, 363202.

PERDEW, J. P. ENZERHOF, M. , BURKE, K. 1996. Generalized Gradient Approximation Made Simple. Phys. Rev. Lett. 77, 3865. 